\title{
A Water-Powered Osmotic Microactuator
}

\author{
Yu-Chuan Su, Liwei Lin, Member, IEEE, and Albert P. Pisano
}

\begin{abstract}
This paper presents a microactuator that utilizes osmosis to produce mechanical actuation without consuming any electrical energy. The microactuator is made of cellulose acetate with cylindrical chamber of 500 to $2000 \mu \mathrm{m}$ in diameter and of 200 to $1000 \mu \mathrm{m}$ in depth. Sodium chloride is chosen as the osmotic driving agent to be placed inside the chamber. A semipermeable diaphragm made of cellulose acetate is processed at the bottom of the chamber to control the water flow. Either a cellulose acetate diaphragm or an impermeable diaphragm made of vinylidene chloride and acrylonitrile copolymer is spin-coated on top of the chamber as the actuation diaphragm. Using the principle of osmosis, this water-powered, osmotic microactuator can employ high osmotic pressure (a chemical potential) up to 35.6 MPa to provide hydrostatic pressure for mechanical actuation. Experimental measurements show that up to $800 \mu \mathrm{m}$ vertical diaphragm movement (diaphragm size of $800 \mu \mathrm{m}$ in diameter) and constant volume expansion rate of 4.5 to $11.5 \mathrm{~nL} / \mathrm{h}$ can be achieved. When integrated with other microfluidic devices, this osmotic microactuator could serve as a clean, compact and inexpensive fluidic actuation source.

Index Terms-microactuator, microfluidics, osmosis, semipermeable membrane.

\section{INTRODUCTION}

$\mathbf{I}$ $\mathrm{N}$ THE past decade, many microactuators based on different physical and chemical principles have been fabricated and investigated for potential applications in microelectromechanical systems (MEMS) [1]. Up to now, most existing microactuators are driven by externally supplied, electric power sources, such as electrostatic motors [2], bubble-powered actuators [3] and electroosmotic pumps [4]. For applications such as implantable and portable devices that electrical power is difficult to obtain, alterative power sources are attractive. Currently, little work has been done in this area.

Osmosis is a passive transport mechanism controlling water flow into and out of cells and is widely known in biological systems [5]. The working principle of osmosis used in this work is based on the water flow from the environment that has lower concentration of osmotic solute to the micromachined chamber that has higher concentration of osmotic solute through a semipermeable diaphragm in order to reach concentration balance. The net flow of water passing through the semipermeable diaphragm into the chamber can generate hydrostatic pressure and push the actuation diaphragm for various applications. Previously, osmosis has been utilized on

Manuscript received November 16, 2001; revised May 2, 2002. This work was supported in part by a DARPA/ MTO/BioFlips grant. This paper was presented in part at the 14th IEEE International Conference on Micro Electro Mechanical Systems. Subject Editor G. B. Hocker.

The authors are with the Department of Mechanical Engineering and Berkeley Sensor and Actuator Center, University of California, Berkeley, CA 94720-1740 USA (e-mail: yuchsu@ me.berkeley.edu).

Digital Object Identifier 10.1109/JMEMS.2002.805045 the macroscale osmotic pumps for drug delivery applications [6], [7]. For example, osmotic pumps with a diameter down to $6 \mathrm{~mm}$ and a minimal length of $15 \mathrm{~mm}$ have been developed and implanted into laboratory animals [8].

When applied to MEMS, osmosis offers attractive features as the actuation principle because it requires no electric power source for operation. Furthermore, the actuation speed is controlled by the characteristics of the semipermeable diaphragm, such as area and permeability, and a constant volume expansion output is expected when the osmotic actuator is operated under saturated state. This paper applies the principle of osmosis to micro devices as the actuation mechanism for potential applications including drug delivery and other biomedical systems. Since no electricity is required, this osmotic actuator could serve as a clean, portable and compact actuation source.

\section{PRINCIPLES AND TheORETICAL MODELS}

Fig. 1 shows the schematic diagram of the osmotic microactuator. Dry osmotic driving agent is packed in a chamber. An actuation diaphragm is constructed on top and a semipermeable diaphragm is constructed on the bottom of the chamber. Water diffuses into the chamber due to osmosis to reduce the concentration difference. The opposite osmotic solute diffusion process is blocked by the semipermeable diaphragm. This results in a net flow of water across the semipermeable diaphragm into the chamber that contains osmotic driving agent. The water flow is controlled by the combination of several factors, including the permeability of the semipermeable diaphragm, the osmotic pressure of the driving agent and the hydrostatic pressure difference across the semipermeable diaphragm. The flow rate of water across the semipermeable diaphragm can be written as the following [7]:

$$
J=K A(\sigma \Delta \pi-\Delta P)
$$

where $J$ is the flow rate of water (volume of water transported per unit time), $K$ is the permeability of the semipermeable diaphragm with respect to water and $A$ is the effective surface area of the semipermeable diaphragm. When water diffuses through the semipermeable diaphragm, the hydrostatic pressure inside the chamber increases and $\Delta P$ is the difference of the hydrostatic pressure across the semipermeable diaphragm that has a negative effect on the flow rate. On the other hand, $\Delta \pi$ is the difference of osmotic pressure across the semipermeable diaphragm that has a positive effect on the flow rate. An osmotic reflection coefficient, $\sigma$, of the semipermeable diaphragm is used to account the outward diffusion of the osmotic driving agent through the semipermeable diaphragm. Ideally, $\sigma$ is equal to 1 and no driving agent will diffuse outward. A net flow of water is able to diffuse through the semipermeable diaphragm into the chamber if the difference of osmotic pressure is higher 


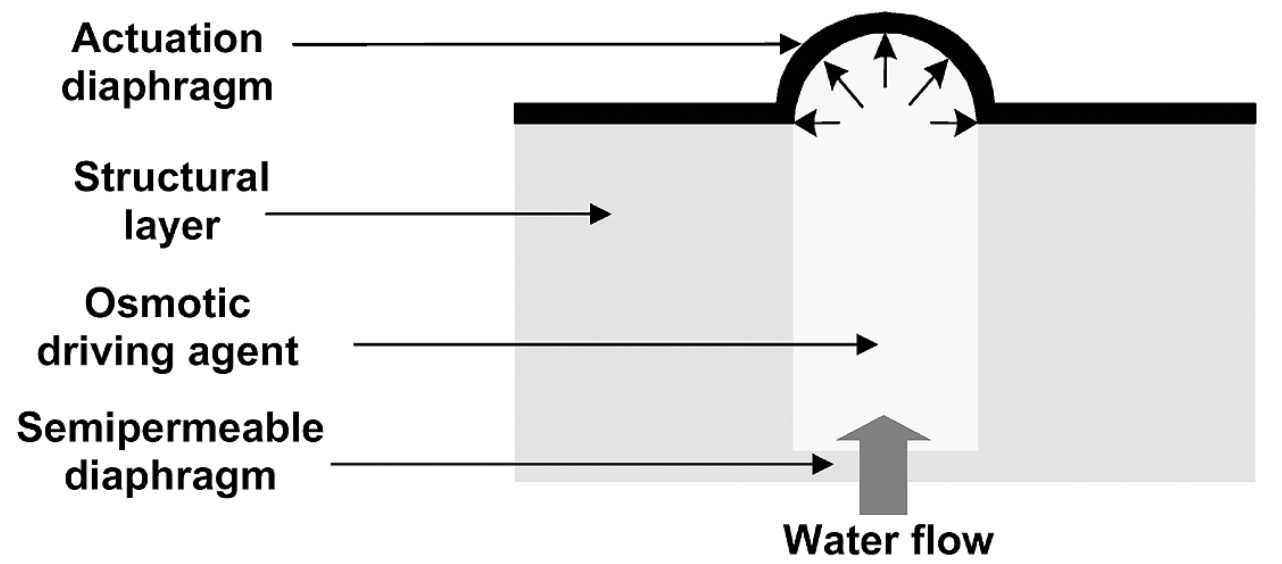

Fig. 1. Schematic of osmotic actuation.

than that of the hydrostatic pressure across the semipermeable diaphragm. The osmotic pressure, a chemical potential, of a solution can be represented as the following [7]:

$$
\pi=S i R T
$$

where $S$ is the solubility of the driving agent in water, $i$ is the number of ions per mole in the solution, $R$ is the ideal gas constant and $T$ is absolute temperature. Driven by the difference of osmotic pressure across the semipermeable diaphragm, water will diffuse into the chamber and increase the hydrostatic pressure inside the chamber such that the actuation diaphragm is forced to deform outwards as shown in Fig. 1. For example, the difference of osmotic pressure is $35.6 \mathrm{MPa}$ when pure water is in contact with saturated sodium chloride solution [9]. In this case, water will keep flowing into the chamber and the actuation diaphragm will keep expanding unless a hydrostatic pressure difference of 35.6 MPa is built across the semipermeable diaphragm to cease the water flow. By using theories of shell and plate, the deformation of the actuation diaphragm can be approximated by the following equation under the condition of small deflection [10]:

$$
w_{0}=0.662 a\left(\frac{q a}{E h}\right)^{1 / 3}
$$

where $w_{0}$ is the maximum deflection at the center of the diaphragm, $a$ and $h$ are the radius and thickness of the diaphragm, respectively. $E$ is Young's modulus and $q$ is the pressure difference across the actuation diaphragm. It is observed that the same actuation displacement can be achieved with lower hydrostatic pressure if the actuation diaphragm has larger diameter, smaller thickness, or lower Young's modulus. The experimental results presented in the work is achieved by keeping the sodium chloride solution in the chamber to be saturated during operation and the difference of osmotic pressure across the semipermeable diaphragm to be much larger than that of the hydrostatic pressure. Therefore, the water flow rate into the chamber can be further simplified as

$$
J=K^{\prime} A \Delta \pi
$$

where $K^{\prime}$ represents the combination of $K$ and $\sigma$. It can be observed from (4) that the water flow rate into the chamber is

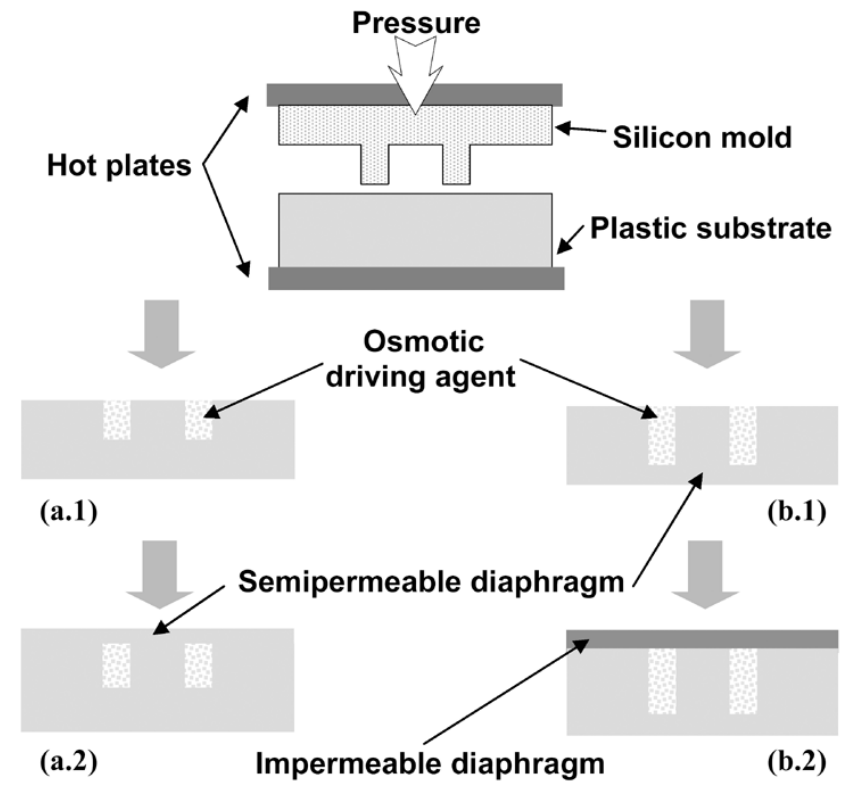

Fig. 2. One-mask fabrication process of the osmotic microactuator (a.1), (a.2) semipermeable-piston type and (b.1), (b.2) impermeable-piston type.

solely controlled by diaphragm properties including the area and permeability of the semipermeable diaphragm with respect to water. When the osmosis pressure difference is a fixed number, the water flow rate will be a constant.

\section{FABRICATION PROCESS}

Two types of osmotic microactuators, including the semipermeable-piston type and the impermeable-piston type, are fabricated by using a one-mask fabrication process as shown in Fig. 2. The semipermeable-piston type actuator uses the same semipermeable diaphragm as both the actuation diaphragm and the semipermeable diaphragm such that water diffuses into the chamber via the actuation diaphragm as shown in Fig. 2(a). The impermeable-piston type actuator uses an impermeable material as the actuation diaphragm and water diffuses into the chamber through the semipermeable diaphragm on the backside of the chamber as shown in Fig. 2(b).

The manufacturing process starts with the fabrication of the silicon mold by using the deep reactive ion etching process. 
Cylindrical columns of 500 to $2000 \mu \mathrm{m}$ in diameter and 200 to $1000 \mu \mathrm{m}$ in height are designed and fabricated on silicon wafers. A micro hot embossing process [11], [12] is followed to make micro chambers on the surface of cellulose acetate sheet. Cellulose acetate is chosen as the material to construct the microactuator because of its semipermeable property. A constant pressure of $0.2 \mathrm{MPa}$ is applied throughout the hot embossing process, including the imprinting stage and the cooling stage, to obtain good replications. The standard process takes about 30 to $50 \mathrm{~min}$ at an elevated temperature of $130^{\circ} \mathrm{C}$ followed by a 15 -min cooling period [13]. If the depth of the chamber is properly designed, this hot embossing process can fabricate semipermeable diaphragm of 150 to $400 \mu \mathrm{m}$ in thickness at the backside of the chamber for the impermeable-piston type actuator as shown in Fig. 2(b.1). However, if the desirable thickness of this backside semipermeable diaphragm is less than $150 \mu \mathrm{m}$, it is difficult to accomplish the requirement by this approach. A thin cellulose acetate diaphragm of desirable thickness can be prefabricated and be solvent-welded on the backside of a cellulose acetate substrate with mechanically-drilled through holes. It is found that ethyl acetate, which dissolves cellulose acetate partially, is suitable as the solvent to bond cellulose acetate films. Sodium chloride powder is then manually placed into the chambers as the osmotic driving agent as shown in Fig. 2(a.1) and (b.1). Any extra salt spilled on the substrate is removed to keep the surface flat and clean. In order to densify the osmotic driving agent, repeated filling, wetting, drying and pressing steps are performed by using the combination of solid osmotic driving agent and saturated solution. In the case of semipermeable-piston actuator, cellulose acetate that plays the dual role of semipermeable and actuation diaphragm is dissolved in acetone and spin-coated on top of the actuator as shown in Fig. 2(a.2). A thickness of $20 \mu \mathrm{m}$ is used such that the diaphragm is permeable to water but not to the osmotic driving agent. For the impermeable-piston type microactuator, an impermeable diaphragm consisting of Saran F-310 solvent-soluble barrier polymer [14] (vinylidene chloride and acrylonitrile copolymer) is employed by spin-on coating as the actuation diaphragm as shown in Fig. 2(b.2). The powder of Saran F-310 resin is first dissolved in the solvent of $65 \%$ methyl ethyl ketone and 35\% toluene. Experimentally, up to $20 \%$ in weight of F-310 powder is added to the solvent and the mixture is kept at $23^{\circ} \mathrm{C}$ for one hour until the $\mathrm{F}-310$ powder is completely dissolved.

Fig. 3 shows the SEM micrographs of silicon mold [see Fig. 3(a)] and molded cellulose acetate substrate [see Fig. 3(b)]. A good replication is illustrated. The cross-sectional views of both semipermeable-piston type and impermeable-piston type microactuators are shown in Fig. 4 where the osmotic driving agent is removed. The semipermeable-piston type microactuator shown in Fig. 4(a) has the actuation diaphragm thickness of $20 \mu \mathrm{m}$, chamber diameter of $1.5 \mathrm{~mm}$ and depth of $500 \mu \mathrm{m}$. The $500-\mu \mathrm{m}$-thick layer at the bottom of the chamber is designed to prevent water penetration during the operation of the actuator. In this case, the top semipermeable diaphragm works for both the purposes of water inlet and osmotic actuation. On the other hand, the impermeable-piston type microactuator shown in Fig. 4(b) uses a semipermeable

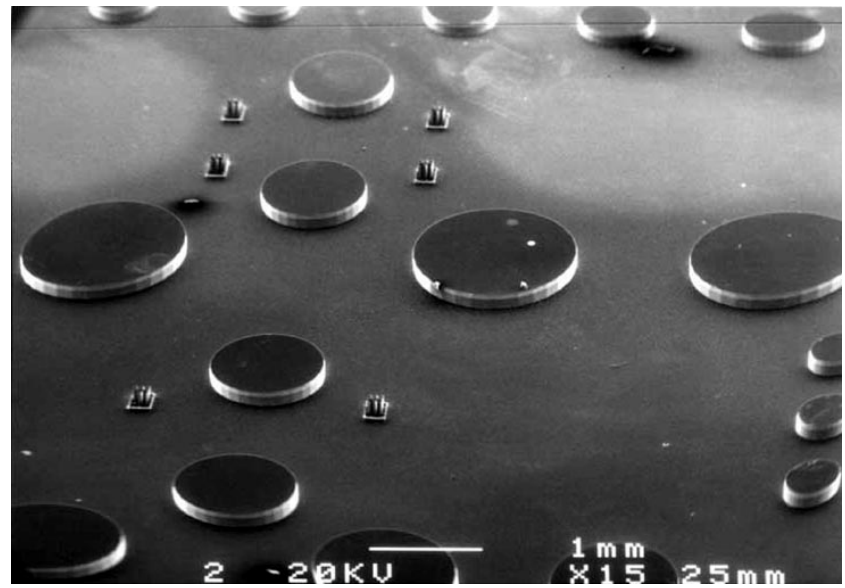

(a)

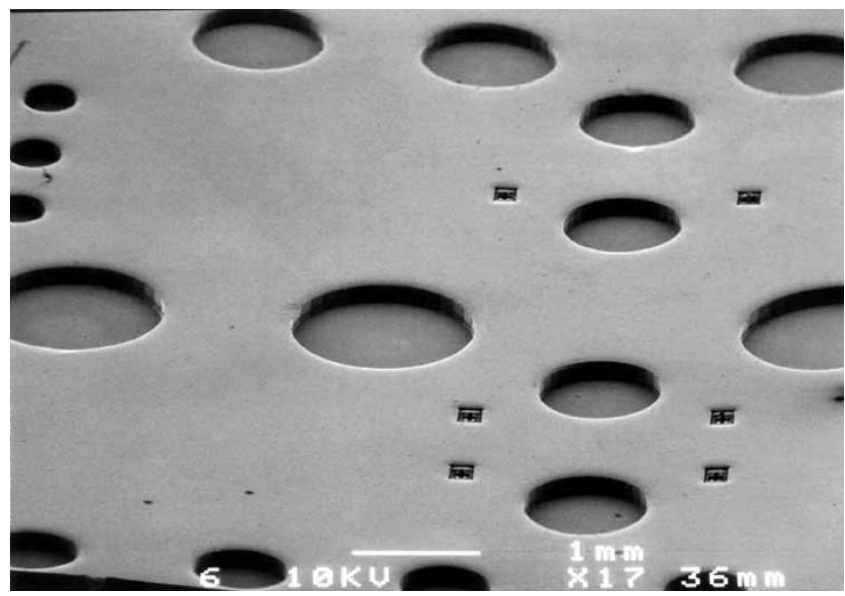

(b)

Fig. 3. SEMs of (a) silicon mold and (b) molded cellulose acetate substrate.

diaphragm with thickness of $100 \mu \mathrm{m}$ and a chamber of $800 \mu \mathrm{m}$ in diameter and $1000 \mu \mathrm{m}$ in depth. The actuation diaphragm is impermeable with thickness of $40 \mu \mathrm{m}$. Fig. 5 shows the SEM micrographs of an impermeable-piston type microactuator before and after the actuation caused by osmosis. Before the actuation, the actuation diaphragm is relatively flat but reflects the uneven surface of osmotic driving agent as shown in Fig. 5(a). After the actuation, a dome shape structure is formed as shown in Fig. 5(b). Fig. 5(c) shows the microactuator that is cut in half after actuation. Delamination of actuation diaphragm at the boundary of chamber is clearly observed. It appears that the effective normal force generated by the hydrostatic pressure difference across the actuation diaphragm will eventually be larger than the bonding strength to cause the separation.

\section{Measurement Results AND Discussions}

The actuation process is observed and the profiles of the actuator at different actuation stages are recorded by tilting the samples $90^{\circ}$ under an optical microscope. Fig. 6 shows the profiles of an actuation diaphragm with an initial diameter of $500 \mu \mathrm{m}$ at six different time steps. The experiment stops after 33 hours when solid osmotic driving agent is completely dissolved. The complete dissolution of solid osmotic driving agent is generally used as the end point of operation in the macroscale osmotic 


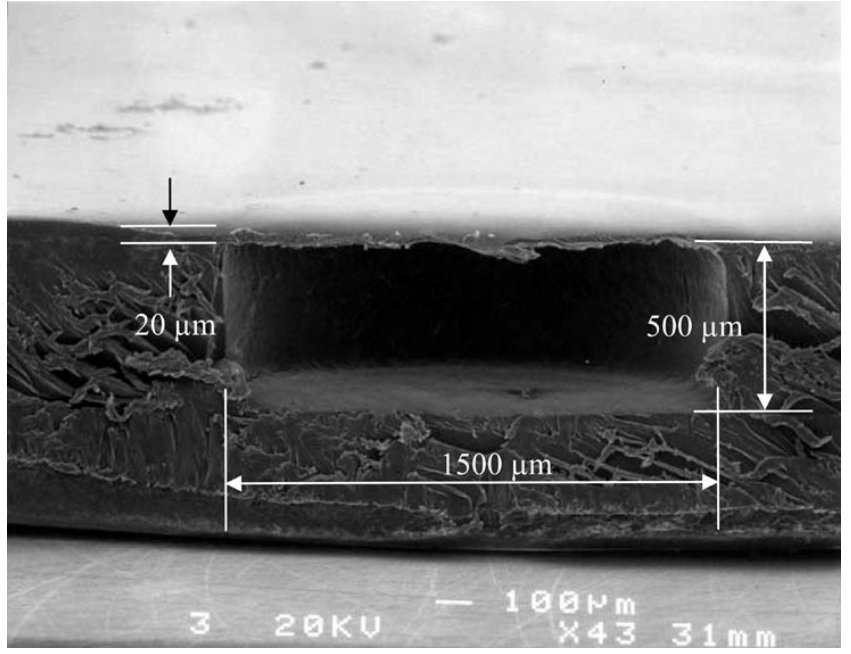

(a)

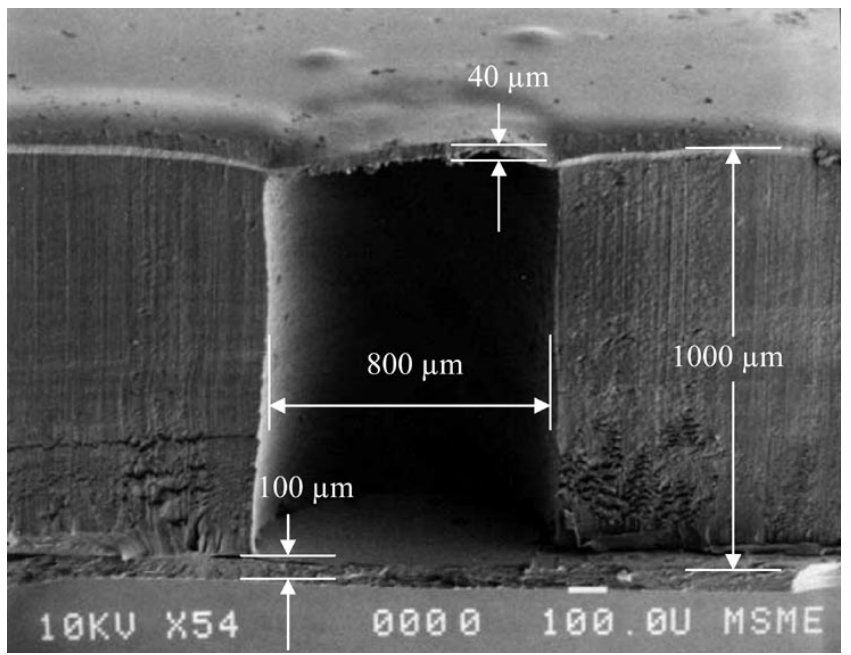

(b)

Fig. 4. Cross sections of (a) a semipermeable-piston type and (b) an impermeable-piston type osmotic microactuators.

pumps [5]. Further operations show that equilibrium across this semipermeable diaphragm will be established eventually and the actuation diaphragm will stop expanding. It is observed that the boundary of the actuation diaphragm is fixed at $250 \mu \mathrm{m}$ initially (only half of the profile is shown due to symmetry). The peak displacement increases continuously as the operation time increases and the actuation diaphragm starts to separate from the substrate after about 1172 minutes into the operation. The volume expansion is calculated by the integration of recorded profiles. Fig. 7 shows the measured peak displacement as well as volume expansion versus operation time of two impermeable-piston-type microactuators of 0.5 and $0.8 \mathrm{~mm}$ in diaphragm diameter and 0.5 and $1 \mathrm{~mm}$ in chamber depth, respectively. The thickness of both actuation diaphragms is $40 \mu \mathrm{m}$ and the thickness of both semipermeable diaphragms is $70 \mu \mathrm{m}$. A delay of actuation of 5 to $10 \mathrm{~h}$ is observed in the beginning as expected. This is the required time for water to penetrate the semipermeable diaphragm and completely fill up the chamber. The magnitude of the time delay depends mainly on the volume of the chamber and the area and water permeability of the semipermeable diaphragm. The measured volume expansion rates of

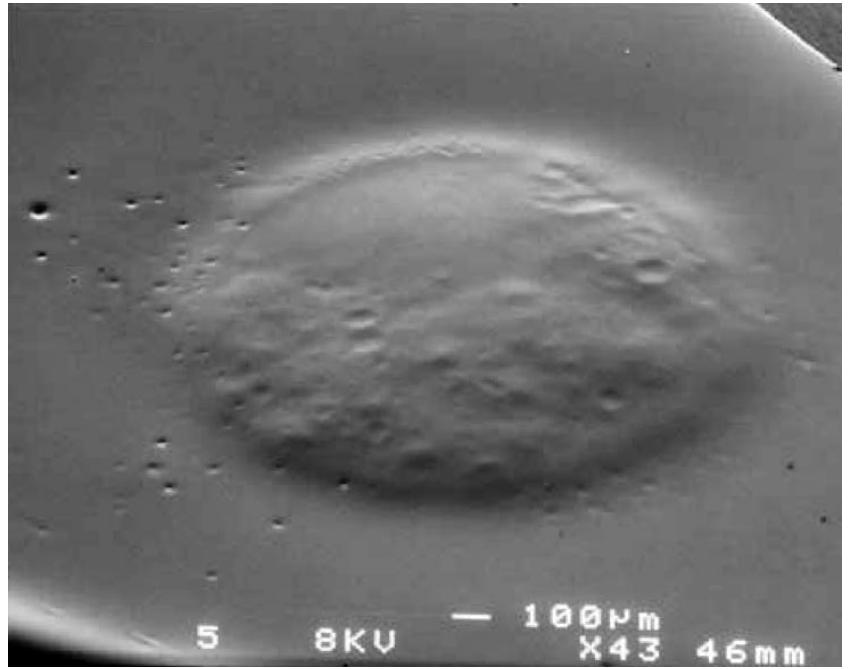

(a)

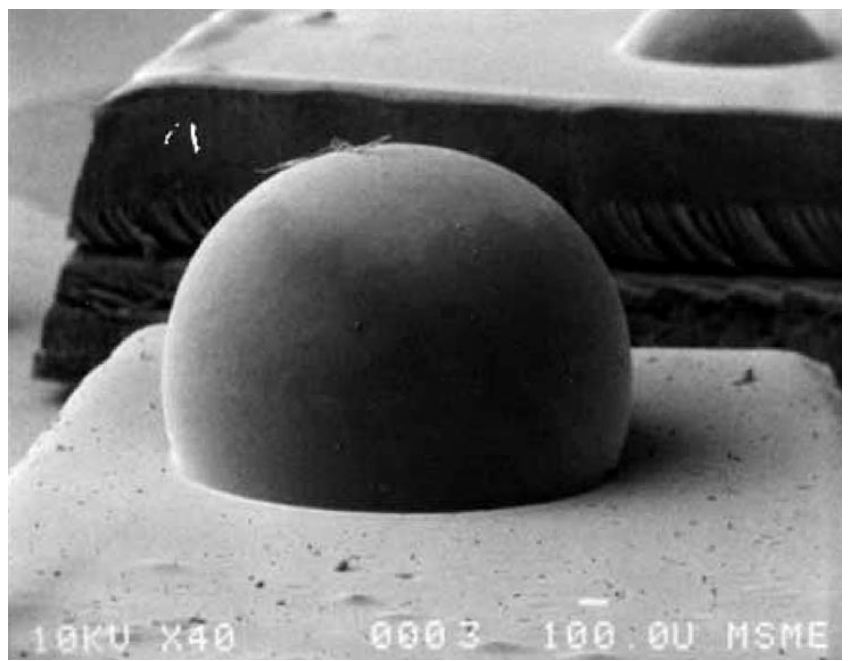

(b)

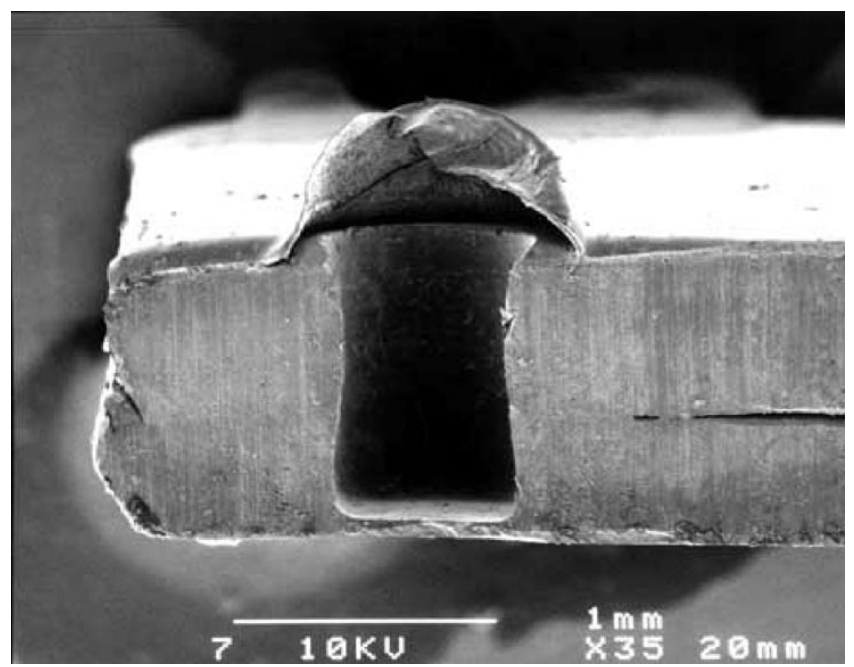

(c)

Fig. 5. SEMs of an impermeable-piston type microactuator showing (a) before and (b) after immersing into water. (c) Cross section of a microactuator after actuation.

the microactuators of 0.5 and $0.8 \mathrm{~mm}$ in diameter are 4.5 and $11.5 \mathrm{~nL} / \mathrm{h}$ and the peak displacements are 400 and $800 \mu \mathrm{m}$, re- 


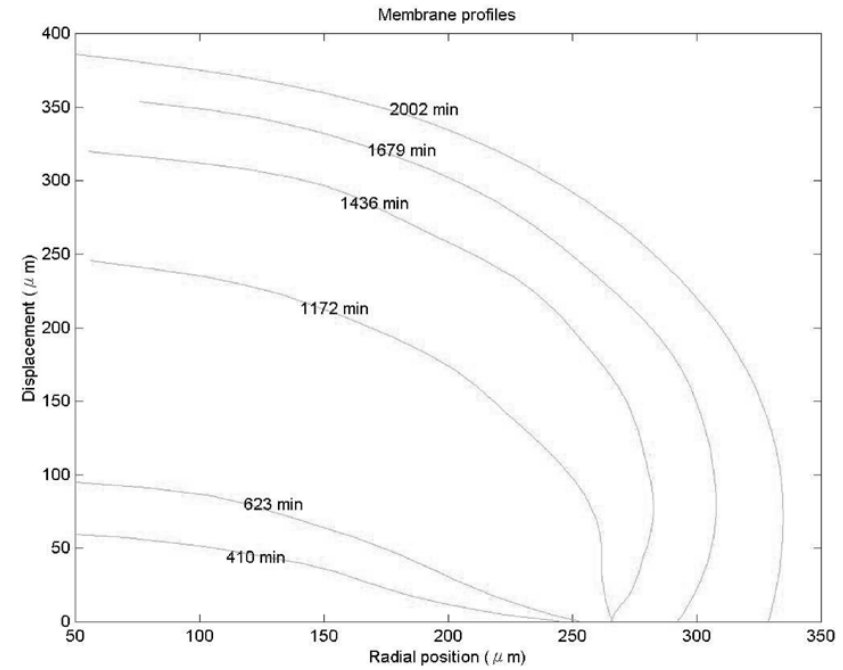

Fig. 6. Measured profiles of an actuation diaphragm during the expansion process.

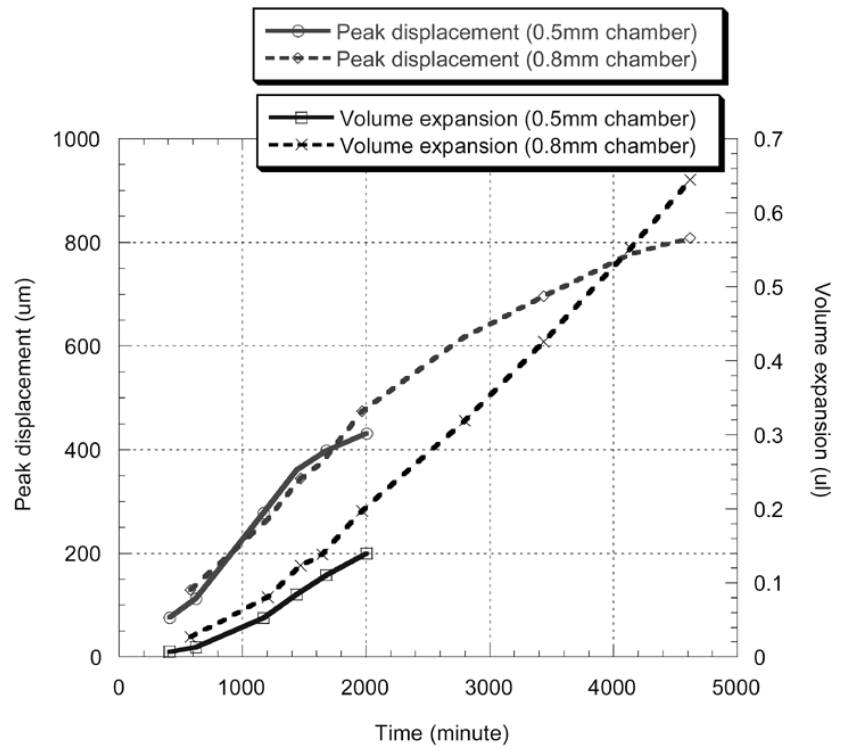

Fig. 7. Measured peak displacement and volume expansion versus actuation time.

spectively. During the last few records of the experiments, the increases of peak displacements slow down while the increases in volume expansion remain constant. Actuators of desired characteristics can be designed and optimized by selecting the right combination of diaphragm dimensions, materials and the right type of osmotic driving agent.

Fig. 8 shows an idealized stress-strain curve for a ductile polymer sample [15]. Initially, the stress is proportional to the strain according to Hooke's law. Based on small deflection operation, the estimated peak displacement at the center of a $0.5 \mathrm{~mm}$ in diameter, $40 \mu \mathrm{m}$ in thickness actuation diaphragm is $4 \mu \mathrm{m}$ by using (3) under a hydrostatic pressure difference of $1 \mathrm{kPa}$. Nonlinear behavior occurs when the deflection of the actuation diaphragm is larger than about $10 \%$ of its thickness and yielding occurs when the strain reaches 5 to $10 \%$ for polymer materials. The stress falls after yielding and settles at a constant value for most polymers. Eventually, strain hardening occurs and the

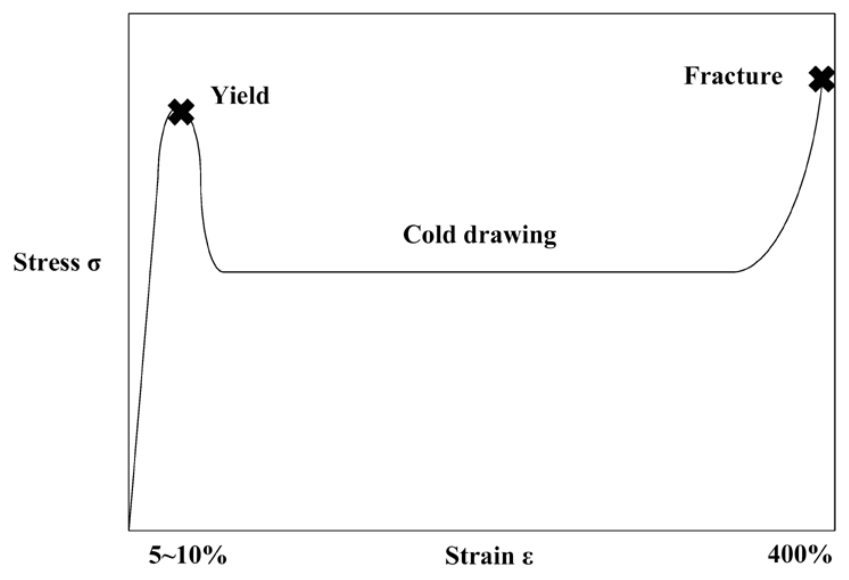

Fig. 8. Idealized stress-strain curve for a ductile polymer sample.

stress rises until fracture. It is desirable to choose materials with low elastic modulus and high fracture strain to increase the expandability of the actuation diaphragm. For example, the impermeable diaphragm, F-310, used in this paper has elastic modulus of less than $100 \mathrm{MPa}$ and fracture strain as high as $400 \%$ [16]. The low elastic modulus, high fracture strain and low permeability to water make Saran F-310 suitable as the actuation diaphragm.

One important phenomenon to be addressed is the initial delay in actuation. From the data shown in Fig. 7, one can extrapolate the starting points of the $0.5-\mathrm{mm}$ and $0.8-\mathrm{mm}$ chamber as 315 and $680 \mathrm{~min}$, respectively. The ratio of the delay time corresponds well to the ratio of the height of the two chambers of 0.5 and $1 \mathrm{~mm}$, respectively. Theoretically, the dissolution of osmotic driving agent in water results in decrease of volume, the electrostriction effect, and decrease of temperature, the endothermic reaction. Experimentally, the maximum solubility of sodium chloride in $100 \mathrm{~g}$ of water at $25^{\circ} \mathrm{C}$ is $35.7 \mathrm{~g}$. Under saturated state, 1 unit volume of water being drawn into the chamber will result in only 0.964 , not 1 full unit of volume increase in the saturated solution because of the electrostriction effect. Since the reduction of $3.6 \%$ in volume increase is small, the electrostriction effect is not the major factor in the initial delay of actuation. The main factor that causes this startup delay is identified as voids existing in the chamber. Because the osmotic driving agent is filled into the chamber in the form of rigid powder, part of the chamber volume will be occupied by air voids initially. Analyzing the data shown in Fig. 7, it is found that roughly $20 \%$ of the chamber volume (occupied by voids initially) needs to be filled with water before diaphragm actuation. This volume percentage of void is the same for the two actuators shown in Fig. 7 since the fabrication process of packing the osmotic driving agent is the same. Furthermore, the semipermeable diaphragms of the two actuators have the same thickness and material properties. Therefore, the delay time should be proportional to the chamber height as measured experimentally. On the other hand, the hydration of sodium chloride also builds a transient temperature variation inside the chamber. The enthalpy of solvation for sodium chloride is $3.88 \mathrm{~kJ} / \mathrm{mol}$ and the positive value indicates an endothermic reaction. The maximum possible temperature drop during the 
actuation in the chamber is estimated to be less than $3^{\circ} \mathrm{C}$ (over the operation period) and should not have any significant effect on the actuation.

When the osmotic actuator is placed in an aqueous environment, a net volume of water diffuses into the chamber and gradually builds up the necessary hydrostatic pressure for the mechanical actuation. Experimentally, the actuator is always operated under saturated state (with dry osmotic driving agent existing in the chamber) with a high osmotic pressure (35.6 MPa) and the required hydrostatic pressure to push the actuation diaphragm is much smaller. Therefore, (1) can be simplified as (4) and the net water flow rate into the chamber will be constant during operation. The volume expansion rate of the actuator will be close but less to the net water inflow rate because of the electrostriction effect. For example, it is observed that the volume expansion rates are constants of 4.5 and $11.5 \mathrm{~nL} / \mathrm{hr}$ for the two actuators presented in Fig. 7. Furthermore, the ratio of these numbers is 2.56 and it is exactly the area ratio of the two actuators $(0.5$ and $0.8 \mathrm{~mm}$ in diameter) as predicted by (4). In addition to the area effect, modifying the composition or reducing the thickness of the semipermeable diaphragm to enhance the permeability to water can further improve the volume expansion rate.

Overall, there are eight parameters and related constraints for the design of osmotic microactuator. The first three parameters are the diameter, thickness and permeability of the semipermeable diaphragm that determine the water flow rate. Among these three, permeability is a material property, which could be chemically or physically modified to achieve targeted value. The diameter and thickness of the semipermeable diaphragm are controlled by design and manufacturing process, respectively. However, desired flow rates must be synthesized under the constraints of structural rigidity to assure that there will be no significant deformation. The fourth parameter is the volume of osmotic driving agent chamber that determines the working period and expansion volume that an osmotic actuator can provide. Enough salt is needed in the osmotic chamber to keep the solution saturated through out the whole actuation period. The next three parameters are the diameter, thickness and permeability of the actuation diaphragm. The diameter of the actuation diaphragm is determined by the constraint of space and the type of application. An actuation diaphragm with minimum thickness is desirable to increase the flexibility of the diaphragm but must be thick enough to block the penetration of water. The final parameter is the osmotic driving agent. Sodium chloride is used in our experiments as the driving agent and its osmotic pressure under saturated state is $35.6 \mathrm{MPa}$ as listed in the reference works. Other chemicals with high osmotic pressure could also be considered as osmotic driving agents in the osmotic actuator applications.

\section{CONCLUSION}

A microactuator that employs osmosis to provide mechanical actuation without consuming electrical energy has been successfully demonstrated. It has been observed that large mechanical displacement can be achieved at the center of a circular-shape actuation diaphragm to about the same distance of its diameter.
When operated under saturated state with fixed manufacturing parameters, the volume expansion rate of the actuator is a constant and is proportional to the area of the semipermeable diaphragm that controls the inflow of water. Experimental results on two fabricated osmotic actuators of 0.5 and $0.8 \mathrm{~mm}$ in diameter show that volume expansion rates of 4.5 and $11.5 \mathrm{~nL} / \mathrm{h}$ are achieved, respectively, and the ratio is proportional to the area of the semipermeable diaphragm as predicted. A time delay in initial actuation is observed as the time required for the beginning of operation and is proportional to the height of the chamber when other parameters are fixed. Experimentally, 315 and $680 \mathrm{~min}$ of delay operation are found on the two fabricated actuators of 0.5 and $1 \mathrm{~mm}$ in height, respectively. The results match the analytical prediction well. The expansion rate and operation characteristics of the actuator can be designed by choosing the right combinations, including material and geometry, of semipermeable diaphragm, impermeable diaphragm and chamber. When integrated with other micro devices, this osmotic actuator could serve as a clean, compact and inexpensive actuation source.

\section{ACKNOWLEDGMENT}

The authors would like to thank ALZA Corporation for providing cellulose acetate solutions and Dow Plastics for providing plastic resins of impermeable materials. The silicon molds are fabricated in the University of California at Berkeley microfabrication laboratory.

\section{REFERENCES}

[1] G. T. A. Kovacs, Micromachined Transducers Sourcebook. New York: McGraw-Hill, 1998.

[2] L. S. Fan, Y. C. Tai, and R. S. Muller, "IC-Processed electrostatic micromotors," Sens. Actuators, vol. 20, pp. 41-47, 1989.

[3] L. Lin and A. P. Pisano, "Thermal bubble powered microactuators," Microsyst. Technol., vol. 1, pp. 51-58, 1994.

[4] A. Manz, C. S. Effenhauser, N. Burggraf, D. J. Harrison, K. Seiler, and K. Flurri, "Electroosmotic pumping and electrophoretic separations for miniaturized chemical analysis systems," J. Micromechan. Microeng., vol. 4, pp. 257-265, 1994.

[5] S. Vogel, Cats' Paws and Catapults. New York: Norton, 1998.

[6] F. Theeuwes, "Elementary osmotic pump," J. Pharmaceut. Sci., vol. 64, pp. 1987-1991, 1975.

[7] F. Theeuwes and S. I. Yum, "Principles of the design and operation of generic osmotic pumps for the delivery of semisolid or liquid drug formulations," Ann. Biomed. Eng., vol. 4, pp. 343-353, 1976.

[8] Alzet Osmotic Pumps [Online]. Available: www.alzet.com

[9] E. Mathiowitz, Encyclopedia of Controlled Drug Delivery. New York: Wiley, 1999.

[10] S. Timoshenko and S. Woinowsky-Krieger, Theory of Plates and Shells. New York: McGraw-Hill, 1959.

[11] L. Lin, Y. T. Cheng, and C. J. Chiu, "Comparative study of hot embossed micro structures fabricated by laboratory and commercial environments," Microsyst. Technol., vol. 4, pp. 113-116, 1998.

[12] L. Lin, T. K. Shia, and C. J. Chiu, "Silicon-processed plastic micropyramids for brightness enhancement applications," J. Micromechan. Microeng., vol. 10, pp. 395-400, 2000.

[13] B. G. Casey, W. Monaghan, and C. D. W. Wilkinson, "Embossing of nanoscale features and environments," Microelectron. Eng., vol. 35, pp. 393-396, 1997.

[14] “SARAN Product Information," Dow plastics.

[15] R. J. Young and P. A. Lovell, Introduction to Polymers. London, U.K.: Chapman and Hall, 1991.

[16] C. A. Harper, Handbook of Plastics, Elastomers and Composites. New York: McGraw-Hill, 1996. 
Yu-Chuan Su received the B.S. and M.S. degrees in power mechanical engineering from the National Tsing Hua University, Taiwan, R.O.C., in 1993 and 1995 , respectively. He is currently working toward the Ph.D. degree in mechanical engineering at the University of California at Berkeley.

His research interests include microactuator, microfluidic chip, and the application of polymers and related fabrication processes to MEMS.

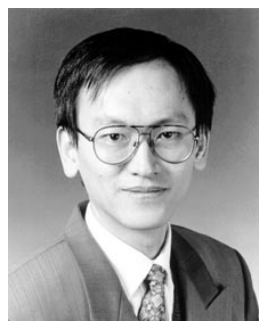

Liwei Lin (S'92-M'93) received the M.S. and Ph.D. degrees in mechanical engineering from the University of California, Berkeley, in 1991 and 1993, respectively.

From 1993 to 1994, he was with BEI Electronics, Inc., in research and development of microsensors. From 1994 to 1996, he was an Associate Professor in the Institute of Applied Mechanics, National Taiwan University, Taiwan. From 1996 to 1999, he was an Assistant Professor at the Mechanical Engineering and Applied Mechanics Department at the University of Michigan. In 1999, he joined the University of California at Berkeley and is now an Associate Professor at Mechanical Engineering Department and Co-Director at Berkeley Sensor and Actuator Center, NSF/Industry/University research cooperative center. His research interests are in design, modeling and fabrication of microstructures, microsensors, and microactuators as well as mechanical issues in microelectromechanical systems including heat transfer, solid/fluid mechanics, and dynamics. He holds seven U.S. patents in the area of MEMS.

Dr. Lin is the recipient of the 1998 NSF CAREER Award for research in MEMS Packaging and the 1999 ASME Journal of Heat Transfer best paper award for his work on microscale bubble formation. He led the effort in establishing the MEMS subdivision in ASME and is currently serving as the Vice Chairman of the Executive Committee for the MEMS subdivision.

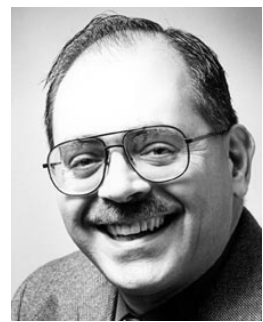

Albert ("Al") P. Pisano is a member of the National Academy of Engineering. He holds the FANUC Chair of Mechanical Systems in the Department of Mechanical Engineering at the University of California at Berkeley and he is jointly appointed to the Department of Electrical Engineering and Computer Science. He is the Director of the Electronics Research Laboratory (administering over $\$ 60$ million in research funds each year) and a Director of the Berkeley Sensor \& Actuator Center (BSAC). His MEMS research includes micropower generation, atomic clocks, drug delivery, RF components, strain sensors, inertial instruments and disk drive actuators. He holds eight patents in MEMS and has coauthored more than 95 journal publications. 